\title{
Polarization resolved intensity noise in vertical-cavity surface-emitting lasers
}

\author{
Josep Mulet, ${ }^{1}$ Claudio R. Mirasso, ${ }^{2}$ and Maxi San Miguel ${ }^{1}$ \\ ${ }^{1}$ Instituto Mediterráneo de Estudios Avanzados, IMEDEA, CSIC-UIB, Universitat de les Illes Balears, \\ E-07071 Palma de Mallorca, Spain \\ ${ }^{2}$ Departament de Física, Universitat de les Illes Balears, E-07071 Palma de Mallorca, Spain
}

(Received 15 January 2001; published 18 July 2001)

\begin{abstract}
We report explicit analytical and numerical results for the polarization resolved intensity noise of verticalcavity surface-emitting lasers operating in the fundamental transverse mode. We describe the fluctuations of the linear and circular polarization components of linearly polarized states on both sides of a nonthermal polarization switching. Our description is valid for small and large birefringence and arbitrary values of the spin flip rate, giving a complete description of the role of these parameters. Normalized cross-correlation functions for both linear and circular components are discussed in detail. They show different degrees of anticorrelated fluctuations in different frequency ranges.
\end{abstract}

DOI: $10.1103 /$ PhysRevA.64.023817

\section{INTRODUCTION}

Mode partition noise (MPN) in semiconductor lasers [1] is detected by means of relative intensity noise (RIN) measurements and gives fundamental information on the dynamical properties of these devices. From the point of view of applications in optical communications, the degradation of the signal to noise ratio associated with MPN fixes limits on receiver sensitivity and bit error rates. Vertical-cavity surface-emitting lasers (VCSELs) operate on a single longitudinal mode, but multitransverse mode operation is common. MPN among these transverse modes and anticorrelated fluctuations of the modes have been described in different experiments and RIN measurements [2-11]. They have also been theoretically characterized [12-14]. The basic physical mechanism for this phenomenon is the same as for MPN among longitudinal modes of edge-emitting lasers, that is, spatial hole burning with modes competing for the same spatial carrier reservoir. The polarization of the light emitted by VCSELs is not as well stabilized as in edge-emitting lasers and VCSELs are known to have a number of polarization instabilities [15]. Transverse modes can have different polarization, but still MPN among different transverse modes is mostly caused by spatial effects.

A more subtle form of MPN occurs in VCSELs operating close to threshold. In this situation VCSELs lase in the fundamental transverse mode, but MPN arises from the competition of the two independent polarization components with essentially the same spatial profile. The effect of polarization fluctuations in the total intensity noise can significantly degrade the RIN characteristics [16] in a system with polarization sensitive elements. The importance of the fluctuations in the polarization component perpendicular to the dominant one has been characterized in detail [17-20]. Evidence of anticorrelated fluctuations of the two polarization components of the fundamental transverse mode has also been reported [20,21].

Due to their high quantum efficiency, low threshold and single mode operation, VCSELs have been proposed as good candidates for the production of quantum squeezed light. In fact, production of squeezed light from VCSELs has been reported by several groups $[5,11,22]$. In this context an im-
PACS number(s): 42.55.Px, 42.65.Pc

portant question that has been addressed is the relevance of polarization partition noise (PPN) in degrading or achieving quantum squeezing [23].

In this paper we study the polarization resolved intensity noise of VCSELs operating in the fundamental transverse mode. We investigate the dynamical origin of anticorrelated polarization fluctuations. Such correlations emerge from mechanisms of polarization coupling and competition that are independent of spatial mode profiles. Anticorrelated dynamics of the polarization components also manifests itself in chaotic regimes caused by optical feedback [24]. We consider here the polarization dynamics within a semiclassical approach. This should give the dynamical understanding necessary for detailed studies of the quantum properties [25].

A standard model for the study of polarization dynamics of VCSELs is the spin flip model (SFM) $[15,26]$ and reduced versions of it [27]. Two important parameters of the model that enter into the description of the dynamical coupling of the two polarization components are the cavity birefringence and the spin flip rate. The latter measures the direct coupling between the two groups of carriers with opposite spin that recombine into photons of opposite circular polarization. Previous studies of polarization fluctuations $[17,20,23]$ take the SFM as a starting point. But, invoking the limit of fast spin flip rate and large birefringence, the SFM is reduced to a simple model with one degree of freedom or to the rate equations for a two-mode laser [1]. However, for VCSELs with small birefringence there is experimental evidence of the role of the nonlinear anisotropies associated with a finite spin flip rate. These effects are seen at least in three different characterizations of polarization fluctuations: A polarization type of four-wave mixing detected in the optical spectrum, polarization resolved intensity noise, and difference in the frequency splitting of the two polarizations at both sides of a polarization switching (PS) [17,19]. In addition, and also for VCSELs with small birefringence, there is evidence of polarization switching [28] caused by phase-amplitude mechanisms of nonthermal origin described by the SFM $[15,29-$ 31]. These results call for the detailed analysis of the complete SFM presented here. Such analysis allows us to gain a full understanding of the dependence of polarization fluctuations on birefringence and spin flip rate in different 
ranges of values of these parameters. Another type of PS that is commonly present in experiments [32,33] arises from the temperature dependence of the gain difference between the two polarization modes, namely, thermal PS. A unified description of the thermal and nonthermal induced PS has recently been introduced in terms of an extended SFM in [31]. In this paper we restrict ourselves to studying the changes in the polarization resolved intensity noise when driving the VCSEL through a nonthermal PS that takes place in the fundamental transverse mode.

Our analysis focuses on the two circularly polarized components of the field. These are the natural variables for the nonlinear dynamics of an active semiconductor material. They are directly phase coupled by the cavity birefringence and also coupled through the carrier populations mixed by spin flip processes. Focusing on the circularly polarized components, we are able to obtain explicit analytical expressions for their RIN spectra (in the approximation of linearized fluctuations). The competing roles of birefringence and spin flip rate become clear from these expressions. Our results for the circularly polarized components are discussed and compared with the polarization resolved RIN of the linearly polarized (LP) components obtained by a numerical analysis.

The paper is organized as follows. The model and some analytical expressions are introduced in Sec. II. In Sec. III we present our results for the polarization resolved intensity noise for the circular and linear components on both sides of a polarization switch. In Sec. IV we discuss the role of birefringence and spin flip rate by visualizing the polarization fluctuations on the Poincare sphere. In Sec. V we give a quantitative description of the anticorrelation of polarization fluctuations for circular and linear polarization components. We analyze the whole range of frequencies, from small frequency to frequencies beyond the relaxation oscillation frequency. Finally, in Sec. VI we give some conclusions.

\section{ANALYSIS OF FLUCTUATIONS}

The SFM [26] assumes a four-level system in which electrons with spin down (up) yield optical transitions with right (left) circularly polarized light $E_{ \pm}$. The SFM rate equations are derived under the basic assumption of fundamental transverse mode operation and constant active region temperature. This model reads

$$
\begin{gathered}
\dot{E}_{ \pm}=\kappa(1+i \alpha)[D \pm d-1] E_{ \pm}-\left(\gamma_{a}+i \gamma_{p}\right) E_{\mp}+F_{ \pm}, \\
\dot{D}=-\gamma(D-\mu)-\gamma(D+d)\left|E_{+}\right|^{2}-\gamma(D-d)\left|E_{-}\right|^{2}-F_{D},
\end{gathered}
$$

$$
\dot{d}=-\gamma_{s} d-\gamma(D+d)\left|E_{+}\right|^{2}+\gamma(D-d)\left|E_{-}\right|^{2}-F_{d} .
$$

$E_{ \pm}$are the two circular components of the electrical field which are related to the linear components by $E_{ \pm}=\left(E_{x}\right.$ $\left.\pm i E_{y}\right) / \sqrt{2}$. The carrier numbers in the two spin channels, i.e., $N_{ \pm}$, are written in terms of the total carrier population $D \equiv\left(N_{+}+N_{-}\right) / 2$ and the carrier difference $d \equiv\left(N_{+}\right.$
$\left.-N_{-}\right) / 2$. The optical field decay rate is $\kappa, \alpha$ is the linewidth enhancement factor, $\gamma$ is the carrier decay rate, $\gamma_{s}$ is the spin flip rate, and the linear anisotropies are $\gamma_{a}$ (dichroism) and $\gamma_{p}$ (birefringence). $\mu$ is the injected current normalized to threshold. For more details about the model and parameters see $[26,29]$.

We have taken into account all noise sources that arise from spontaneous emission processes. These noise sources, which are derived after adiabatic elimination of the material polarization [26], read

$$
\begin{gathered}
F_{ \pm}(t)=\sqrt{\beta_{s p} \gamma(D \pm d)} \xi_{ \pm}(t), \\
F_{\left(\begin{array}{c}
D \\
d
\end{array}\right)}(t)=\frac{\gamma}{2 \kappa}\left[\sqrt{\beta_{s p} \gamma(D+d)} E_{+} \xi_{+}^{*}(t)\right. \\
\left.\quad \pm \sqrt{\beta_{s p} \gamma(D-d)} E_{-} \xi_{-}^{*}(t)+\text { c.c. }\right],
\end{gathered}
$$

where $\beta_{s p}$ is the fraction of the spontaneously emitted photons that goes into the lasing mode. Accordingly, the spontaneous emission rate is $R_{s p}=4 \beta_{s p} \gamma D_{0}, D_{0}$ being the degree of inversion. $\xi_{ \pm}$are two independent complex noise sources with zero mean and correlation $\left\langle\xi_{ \pm}(t) \xi_{ \pm}^{*}\left(t^{\prime}\right)\right\rangle=2 \delta\left(t-t^{\prime}\right)$. Within our semiclassical description we have neglected pump fluctuations, i.e., $\delta \mu(t)=0$.

In the absence of noise, an arbitrary monochromatic solution of Eqs. (1a) $-(1 \mathrm{c})$ can be written as $E_{ \pm}(t)$ $=Q e^{i\left(\omega_{ \pm} t \pm \psi\right)}, D(t)=D_{0}$, and $d(t)=d_{0}$. For an $\hat{x}$-LP state $\psi=0, \omega_{ \pm}=-\gamma_{p}+\alpha \gamma_{a}, D_{0}=1+\gamma_{a} / \kappa$. For a $\hat{y}$-LP state $\psi=\pi / 2, \omega_{ \pm}=\gamma_{p}-\alpha \gamma_{a}, D_{0}=1-\gamma_{a} / \kappa$. In both cases, $d_{0}$ $=0$ and $Q^{\overline{2}}=\left(\mu-D_{0}\right) /\left(2 D_{0}\right)$. A linear stability analysis around these LP states was performed in [29] and discussed in [15]. The polynomial for the complex eigenvalues split into two parts: a second order polynomial that takes account of the total power fluctuations and a third order polynomial that gives information about the polarization stability. The sixth eigenvalue is zero and is associated with the invariance in the field phase orientation. The frequency and damping of the relaxation oscillations (ROs), i.e., $\lambda_{ \pm}=-\Gamma_{R} \pm i \Omega_{R}$, can be obtained from the second order polynomial. Their expressions are

$$
\lambda_{ \pm}=-\frac{\gamma \mu}{2 D_{0}} \pm i \sqrt{2 \kappa \gamma\left(\mu-D_{0}\right)-\left(\frac{\gamma \mu}{2 D_{0}}\right)^{2}}
$$

We have to note that under multitransverse mode operation additional $\mathrm{RO}$ frequencies of the total intensity associated with different transverse modes appear [34]. For the third order polynomial we have

$$
\begin{aligned}
\mathcal{D}(\lambda)= & \lambda^{3}+\left(\frac{\gamma_{s}}{\gamma}+2 Q^{2}-4 \varepsilon \frac{\gamma_{a}}{\gamma}\right) \lambda^{2}+4\left[\left(\frac{\gamma_{p}}{\gamma}\right)^{2}+\left(\frac{\gamma_{a}}{\gamma}\right)^{2}\right. \\
& \left.+\frac{\kappa}{\gamma} Q^{2} D_{0}-\varepsilon \frac{\gamma_{a}}{\gamma}\left(\frac{\gamma_{s}}{\gamma}+2 Q^{2}\right)\right] \lambda+4\left[\left(\frac{\gamma_{p}}{\gamma}\right)^{2}+\left(\frac{\gamma_{a}}{\gamma}\right)^{2}\right] \\
& \times\left(\frac{\gamma_{s}}{\gamma}+2 Q^{2}\right)-8 \varepsilon \frac{\kappa}{\gamma} Q^{2} D_{0}\left(\frac{\gamma_{a}}{\gamma}+\alpha \frac{\gamma_{p}}{\gamma}\right)
\end{aligned}
$$


where $\varepsilon=1(-1)$ when a $\hat{x}$ - $(\hat{y}-)$ LP solution is considered. $\mathcal{D}(\lambda)=0$ determines the polarization stability, i.e., for $\operatorname{Re} \lambda$ $>0(<0)$ the state is unstable (stable). PS takes place as a consequence of a change in the stability of the polarization states, for instance, increasing the injection current. Both $\hat{x}$ LP and $\hat{y}$ LP states are stable below PS (coexistence regime) except for currents close to threshold, due to the presence of $\gamma_{a}$. Above the PS only $\hat{y}$ is stable. Therefore, a nonthermally induced PS from the low frequency $(\hat{x})$ to the high frequency $(\hat{y})$ mode takes place.

Analyzing the solutions of Eq. (4), damped oscillations can appear in different regimes of parameters [15]. We consider the situation where $d(t)$ relaxes exponentially with time constant $\gamma_{s}^{-1}$ while coupled oscillations (COs) of the ellipticity and polarization orientation appear at frequency $\Omega_{P}$. We can derive an approximate expression for the CO frequency $\Omega_{P} \equiv|\operatorname{Im} \lambda|$ in the limit of large $\gamma_{s}$ given by

$$
\Omega_{P}=2 \gamma_{p}-\varepsilon \alpha \kappa\left(\frac{\mu-D_{0}}{D_{0}}\right) \frac{\gamma}{\gamma_{s}}+o\left(\frac{\gamma}{\gamma_{s}}\right)^{2},
$$

which for $\gamma_{a}=0$ reduces to $\Omega_{n l} \equiv \Omega_{P}-2 \gamma_{p} \approx-\varepsilon \alpha \kappa \gamma(\mu$ $-1) / \gamma_{s}, \Omega_{n l}$ being the nonlinear contribution of the birefringence [18]. In contrast to ROs, the $\mathrm{CO}$ frequency varies linearly with the distance to threshold. From the last expression, the $\Omega_{P}$ frequency can be identified with the birefringence splitting although modified by the spin flip rate. It can be seen that the nonlinear anisotropies defined in $[18,19]$ coincide, at first order in $\gamma / \gamma_{s}$, with those predicted by Eq. (4).

In order to better understand the power fluctuations, Eqs. (1a) $-(1 \mathrm{c})$ can be translated from the field description $E_{ \pm}(t)$ to equations for the intensities $P_{ \pm}(t)$ and the phase differences $\phi(t) \equiv \phi_{+}(t)-\phi_{-}(t)$ by $E_{ \pm}(t)=\sqrt{P_{ \pm}(t)} e^{i \phi_{ \pm}(t)}$. The stochastic transformation in the Itô sense [35] of Eqs. (1a) (1c) reads

$$
\begin{aligned}
\dot{P}_{ \pm}= & 2 \kappa[D \pm d-1] P_{ \pm}-2 \sqrt{P_{+} P_{-}}\left[\gamma_{a} \cos \phi \pm \gamma_{p} \sin \phi\right] \\
& +2 \beta_{s p} \gamma[D \pm d]+F_{P_{ \pm}}, \\
\dot{\phi}= & 2 \kappa \alpha d-\sqrt{\frac{P_{-}}{P_{+}}}\left[\gamma_{p} \cos \phi-\gamma_{a} \sin \phi\right]+\sqrt{\frac{P_{+}}{P_{-}}}\left[\gamma_{p} \cos \phi\right. \\
+ & \left.\gamma_{a} \sin \phi\right]+F_{\phi}, \\
\dot{D}= & -\gamma(D-\mu)-\gamma(D+d) P_{+}-\gamma(D-d) P_{-}-F_{D}, \\
& \dot{d}=-\gamma_{s} d-\gamma(D \mathrm{c})
\end{aligned}
$$

with the Langevin terms

$$
F_{P_{ \pm}}=\sqrt{4 \beta_{s p} \gamma(D \pm d) P_{ \pm}} \xi_{P_{ \pm}},
$$

$$
F_{\phi}=\sqrt{\frac{\beta_{s p} \gamma(D+d)}{P_{+}}} \xi_{\phi_{+}}+\sqrt{\frac{\beta_{s p} \gamma(D-d)}{P_{-}}} \xi_{\phi_{-}},
$$

$$
F_{(D)}=\frac{\gamma}{\kappa}\left[\sqrt{\beta_{s p} \gamma(D+d) P_{+}} \xi_{P_{+}} \pm \sqrt{\beta_{s p} \gamma(D-d) P_{-}} \xi_{P_{-}}\right]
$$

$\xi_{P_{ \pm}}, \xi_{\phi_{ \pm}}$being real white Gaussian random numbers with zero mean and correlation $\left\langle\xi_{a}(t) \xi_{b}\left(t^{\prime}\right)\right\rangle=\delta_{a, b} \delta\left(t-t^{\prime}\right)$.

In order to calculate the power fluctuations of the total intensity and circular components, we linearize Eqs. (6a)(6d) around their steady states. We have $P_{ \pm_{0}}=Q^{2}, d_{0}=0$, $D_{0}=1+\varepsilon \gamma_{a} / \kappa$, and $\phi_{0}=0, \pi$. For convenience, we calculate the fluctuations for the total intensity $\delta P(t)=\delta P_{+}(t)$ $+\delta P_{-}(t)$ and the intensity difference $\delta q(t)=\delta P_{+}(t)$ $-\delta P_{-}(t)$. The linearized equations can be straightforwardly solved via Fourier transform, yielding the expressions

$$
\begin{gathered}
\widetilde{\delta} P(\omega)=\sqrt{2 Q^{2} R_{s p}} \frac{[i \omega+\gamma] \widetilde{\xi}_{P}}{\left[\omega-\Omega_{R}+i \Gamma_{R}\right]\left[\omega+\Omega_{R}+i \Gamma_{R}\right]}, \\
\widetilde{\delta} q(\omega)=\sqrt{2 Q^{2} R_{s p}} \frac{\left[4 \varepsilon \alpha Q^{2} \gamma \gamma_{p}+\left(i \omega-2 \varepsilon \gamma_{a}\right)\left(\gamma_{s}+i \omega\right)\right] \widetilde{\xi}_{q}-2 \varepsilon \gamma_{p}\left(\gamma_{s}+2 \gamma Q^{2}+i \omega\right) \widetilde{\xi}_{\Phi}}{\mathcal{D}(i \omega / \gamma) \gamma^{3}}
\end{gathered}
$$

where $\Gamma_{R}$ and $\Omega_{R}$ are given by Eq. (3) and $\mathcal{D}(\lambda)$ is given by Eq. (4). The Fourier transformed noise sources $\widetilde{\xi}_{P}(\omega)$, $\widetilde{\xi}_{q}(\omega)$, and $\widetilde{\xi}_{\Phi}(\omega)$ verify that $\left\langle\widetilde{\xi}_{i}(\omega) \widetilde{\xi}_{j}(\omega)\right\rangle=\delta_{i, j}$ for $j$ $=P, q, \Phi$.

Let $P_{u}$ and $P_{v}$ be the power of each orthogonal component $(u=+, v=-$ for the circular components or $u=x, v$ $=y$ for the linear components). The power spectrum of each component is defined by

$$
S_{u, v}=\left\langle\left|\widetilde{\delta P}_{u, v}(\omega)\right|^{2}\right\rangle,
$$

while the power spectrum for the total intensity is

$$
S_{P}=\left\langle\left|\widetilde{\delta P}_{u}(\omega)+\widetilde{\delta P}_{v}(\omega)\right|^{2}\right\rangle
$$


$\langle\cdot\rangle$ means the average over different noise realizations. Power spectra can be straightforwardly derived from Eqs. (8a) and (8b) yielding

$$
\begin{gathered}
S_{P}(\omega)=2 Q^{2} R_{s p} \frac{\left(\omega^{2}+\gamma^{2}\right)}{\left[\left(\omega-\Omega_{R}\right)^{2}+\Gamma_{R}^{2}\right]\left[\left(\omega+\Omega_{R}\right)^{2}+\Gamma_{R}^{2}\right]}, \\
S_{q}(\omega)=2 Q^{2} R_{s p} \frac{\left[4 \varepsilon \alpha Q^{2} \gamma \gamma_{p}-2 \varepsilon \gamma_{a} \gamma_{s}-\omega^{2}\right]^{2}+\omega^{2}\left(\gamma_{s}-2 \varepsilon \gamma_{a}\right)^{2}+\left(2 \gamma_{p}\right)^{2}\left[\omega^{2}+\left(\gamma_{s}+2 \gamma Q^{2}\right)^{2}\right]}{\mathcal{D}(i \omega / \gamma) \mathcal{D}(-i \omega / \gamma) \gamma^{6}} .
\end{gathered}
$$

We note that the relative intensity noise is defined as the power spectrum divided by the square of the mean value of the total power, $\bar{P}^{2}=\left(2 Q^{2}\right)^{2}$. The power spectrum of the circular components $S_{ \pm}$can be obtained from Eqs. (11a), and (11b) by taking into account that the fluctuations $\widetilde{\delta P}$ and $\widetilde{\delta q}$ are independent,

$$
S_{ \pm}=\left\langle\left|\widetilde{\delta P}_{ \pm}\right|^{2}\right\rangle=\frac{1}{4}\left(S_{P}+S_{q}\right) .
$$

It is important to remark that the contribution to the noise of the circular components arises from the linear superposition of the total intensity noise $S_{P}$ and the polarization fluctuations $S_{q}$. This separation is possible in LP states because the total intensity and intensity difference fluctuations decouple (at first order). However, for other states (elliptical [36] and dynamical states [29]) the decoupled description is not valid requiring, in principle, the study of a five-dimensional system.

\section{RIN THROUGH A NONTHERMAL POLARIZATION SWITCH}

In this section we present analytical and numerical investigations of the polarization resolved intensity noise. Since the circular basis is the natural basis to study the optical transitions, in Sec. III A we investigate power fluctuations of the circular components while in Sec. III B we discuss the power spectra of the linear components for LP states when crossing a nonthermal polarization switching.

\section{A. Fluctuations of the circular components of LP states}

Two mechanisms are responsible for the coupling between dynamical variables. On one hand, the carriers with spin up and down are coupled through the spin flip rate, and the limits $\gamma_{s} \rightarrow \infty$ and $\gamma_{s} \rightarrow 0$ correspond to the fast spin relaxation and slow spin relaxation rate, respectively. The second mechanism is the birefringence $\gamma_{p}$. Microscopically, the birefringence transforms left polarized photons into right and vice versa. The macroscopic effect is to provide LP states with a well defined polarization orientation. Therefore, we expect that both the spin flip rate and the birefringence play an important role in the origin of noise anticorrelations of the polarized components. Finally, dichroism introduces different losses between the two LP states, selecting the mode with highest (unsaturated) gain at threshold. Measurements of fluctuations of the circular components can be performed by using $\lambda / 4$ plate techniques [18]. We concentrate our study on finite values of the spin flip rate and relatively small birefringence. We investigate the power spectra for different values of the spin flip rate and birefringence while maintaining the rest of the laser parameters fixed: $\gamma=1 \mathrm{~ns}^{-1}, \quad \kappa$ $=300 \mathrm{~ns}^{-1}$, the spontaneous emission coefficient $\beta_{s p}$ $=10^{-5}$, and $\alpha=3$. The dichroism is set to $\gamma_{a} \lesssim 0$ in order to select the low frequency mode $(\hat{x})$ at threshold. A nonthermal PS takes places from the low frequency mode $(\hat{x}$-LP) to the high frequency mode $(\hat{y}$-LP) when the injection current is increased. The characterization of thermally induced and nonthermal PS was discussed in [31,37].

In Fig. 1 we plot the power spectra obtained for $\gamma_{s}$ $=100 \mathrm{~ns}^{-1}, \gamma_{p}=1 \mathrm{~ns}^{-1} ; \mu=1.04<\mu_{s w}$ in Fig. 1(a,b), while $\mu=1.5>\mu_{s w}$ in Fig. 1(c,d). Analytical results obtained from Eqs. (9)-(12) are plotted in Fig. 1(a,c) with solid thin lines. As can be seen, they are in very good agreement with the numerical results. The spectrum of the total intensity has a single peak located at the relaxation oscillation frequency $\nu_{R}=\Omega_{R} /(2 \pi)$. This peak is due to fluctuations in the total photon number. In contrast, the power spectra of the circular components concide and display an additional peak at the CO frequency $\nu_{P}$, which moves toward $\gamma_{p} / \pi$ when $\gamma_{s}$ $\rightarrow \infty$, in agreement with Eq. (5). We note that the height of the CO peak is larger before PS and it appears at lower frequency in qualitative agreement with Ref. [18]. It can be
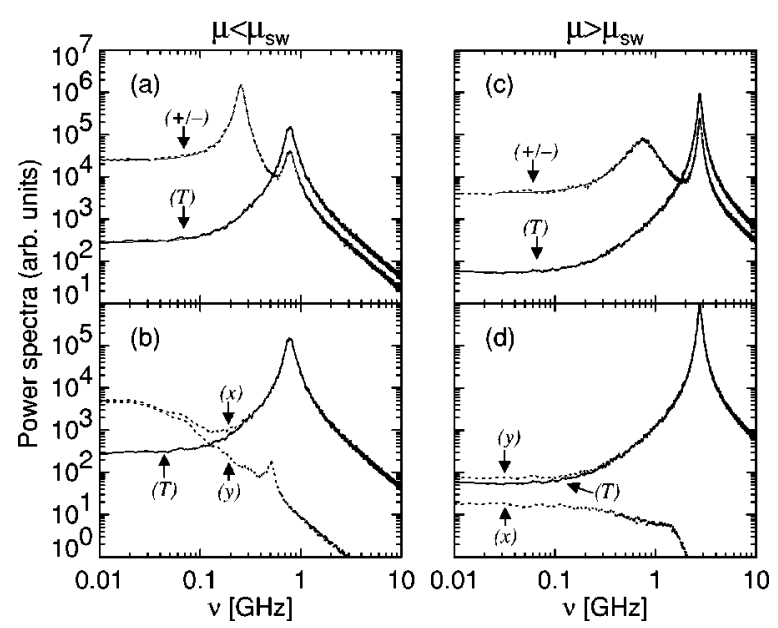

FIG. 1. Power spectra in arbitrary units for currents below PS (a), (b), and above PS (c), (d). Solid thin lines in (a) and (c) represent the theoretical predictions given by Eqs. (11a) and (12). 
clearly seen that the noise in the two circular components is much larger than the total intensity noise at low frequencies, a sign of anticorrelation between $P_{+}$and $P_{-}$. This anticorrelation is interrupted at higher frequencies due to the peak associated with the fluctuations in the total intensity. In fact, we find maximum correlation at $\nu_{R}$, as will be discussed later.

\section{B. Fluctuations of the linear components of LP states}

In this section, we present the power spectra of the linear components corresponding to LP states. When a LP state is considered, there is a linear component that captures nearly the total intensity (lasing component, $\bar{P}_{l}$ ) and another one with very small intensity (nonlasing component $\bar{P}_{n l}$ ). Although a LP state is considered, we find that fluctuations in the nonlasing component are relevant. The power spectrum of the total intensity results from the superposition of the fluctuations of the lasing and nonlasing components.

The power spectrum of the total intensity and the lasing component has a peak located at the RO frequency, as can be seen in Figs. 1(b,d). However, the CO peak is not present in this case. While the lasing component captures nearly all the noise at high frequency, the power spectrum of the nonlasing component has a small peak close to the frequency beating between $\Omega_{R}$ and $\Omega_{P}$. The power spectrum of the nonlasing component has a small contribution to the total noise at high frequencies, displaying a clear Lorentzian decay [20]. The behavior at low frequencies requires a more subtle study. The noise in the two polarization components can exceed the total noise by several orders of magnitude at low frequencies. This particular behavior occurs in LP states with important polarization fluctuations, yielding relative high values of the parameter $M \equiv \bar{P}_{n l} / \bar{P}_{l}$. Below the PS, in the coexistence regime, strong anticorrelated fluctuations appear at low frequencies [Fig. 1(b)]. On the contrary, above the PS, $M$ decreases and anticorrelation nearly vanishes [Fig. 1(d)]. PPN has been claimed to be sensitive to the parameter $M$ [1]. An approximate expression for $M$ was given in Ref. [18] using a one-dimensional version of the SFM based on a Kramers problem.

\section{ROLE OF SPIN FLIP AND BIREFRINGENCE}

Since our theoretical description is valid for small and large values of the birefringence and arbitrary values of the spin flip, in this section we give a complete description of the role of these parameters. We look at the power spectra while the polarization state is followed on the Poincare sphere.

Power spectra for small and large values of $\gamma_{s}$, in absence of birefringence, are shown in Fig. 2. As expected, the $\mathrm{CO}$ peak is absent in the power spectra of the circular components Fig. 2(a,d). The main difference between the two cases appears at low frequency: while $P_{ \pm}$have large anticorrelation for large $\gamma_{s}$, this anticorrelation is reduced for small $\gamma_{s}$. This effect can be understood as follows: for slow spin flip rates each of the two circular components burns carriers from its own reservoir $N_{ \pm}$separately. In this case there is no competition and therefore small anticorrelated fluctuations
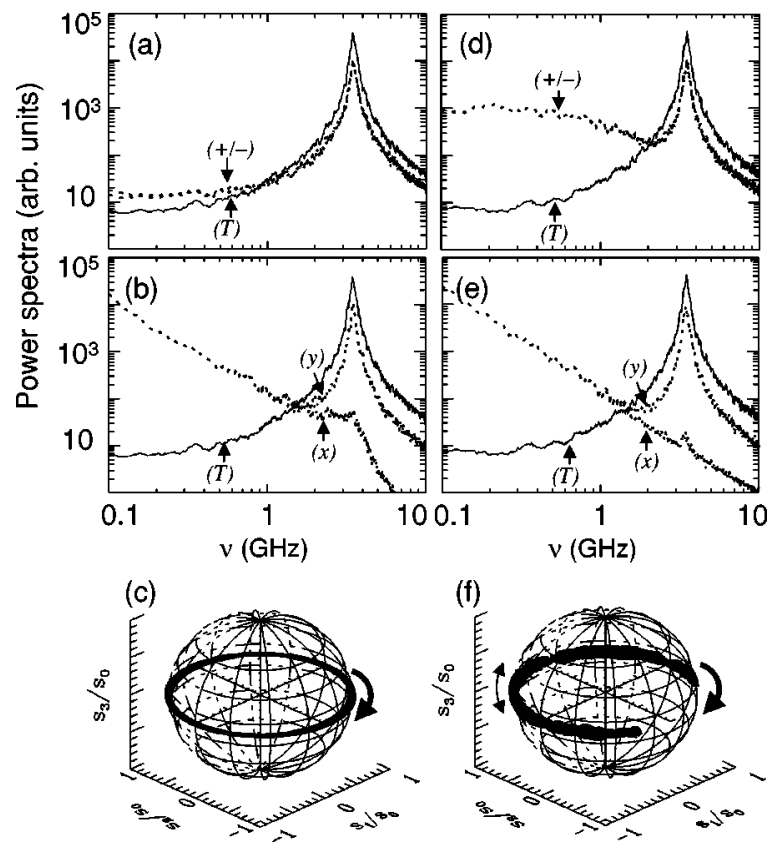

FIG. 2. Study of the effect of $\gamma_{s}$ in the absence of birefringence, in the power spectra of the circular $(\mathrm{a}, \mathrm{d})$ and linear components (b,e). Evolution of the polarization state on the Poincare sphere (c,f) for small values of the spin flip rate $\gamma_{s}=10 \mathrm{~ns}^{-1}$ in $(\mathrm{a}-\mathrm{c}), \gamma_{s}$ $=100 \mathrm{~ns}^{-1}$ in $(\mathrm{d}-\mathrm{f})$. The normalized current is $\mu=1.8$. The meaning of the symbols is $(T)=S_{P},(+/-)=S_{ \pm},(x)=S_{x}$, and $(y)$ $=S_{y}$.

are observed in Fig. 2(a). On the other hand, for fast spin flip rates the two circular components have to share almost the same carrier reservoir since $N_{+} \approx N_{-}$. The latter causes strong anticorrelation because of PPN [Fig. 2(d)]. The power spectra of the two linear components are similar for small and large values of $\gamma_{s}$ [Fig. 2(b,e)]. They show pronounced anticorrelations at low frequencies linked to important changes in the polarization orientation. Both lasing and nonlasing power spectra display a peak at the relaxation oscillation frequency.

The role of the birefringence is shown in Fig. 3 for a fixed value of the spin flip rate. For small birefringence, $\gamma_{p}$ $=0.1 \mathrm{~ns}^{-1}$, we observe large anticorrelation of circular and linear components at low frequencies [Fig. 3(a,b)]. This fact indicates important polarization fluctuations. The main role of the birefringence is to fix a polarization orientation, consequently reducing the polarization fluctuations. For a larger birefringence, $\gamma_{p}=10 \mathrm{~ns}^{-1}$, we observe that the anticorrelation of the circular components has been considerably reduced [Fig. 3(d)], being negligible for the linear components [Fig. 3(e)]. We note that a PS occurs when the birefringence is increased and the fluctuations on the Poincare sphere move to the opposite direction on the equator of the sphere. In addition, the $\mathrm{CO}$ peak appears at larger frequency than the RO peak.

It is also illustrative to analyze the evolution of the polarization state on the Poincare sphere (Fig. 4). The Stokes parameters $S_{j}$ are defined by the following relationships:

$$
S_{0}=\left|E_{+}\right|^{2}+\left|E_{-}\right|^{2}=\left|E_{x}\right|^{2}+\left|E_{y}\right|^{2}=P,
$$



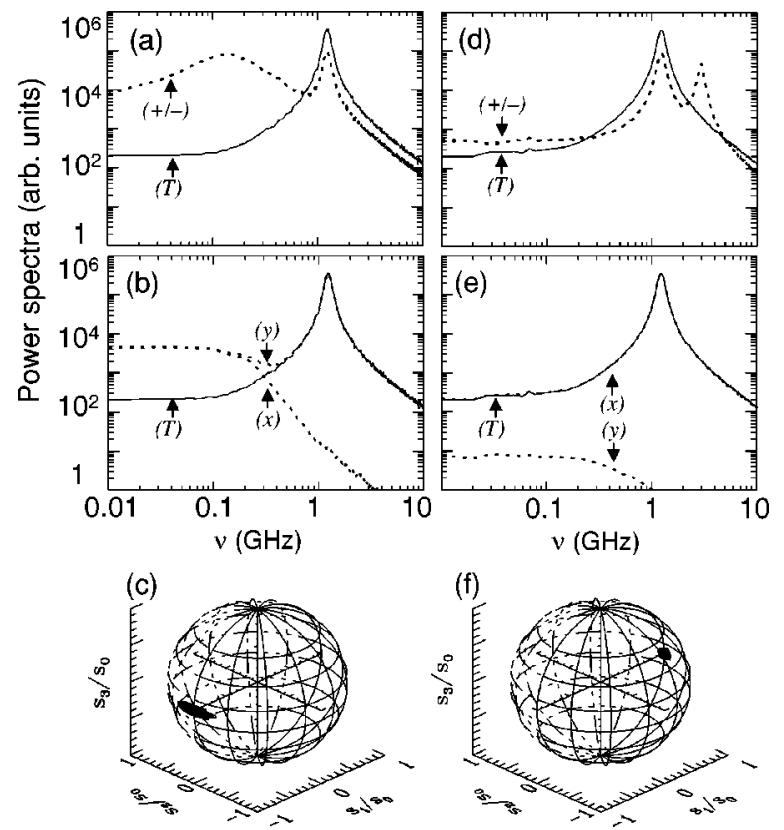

FIG. 3. Study of the effect of $\gamma_{p}$ on power spectra of the circular (a,d) and linear (b,e) components when $\gamma_{s}=50 \mathrm{~ns}^{-1}$. Evolution on the Poincaré sphere for small birefringence $\gamma_{p}=0.1 \mathrm{~ns}^{-1}(\mathrm{a}-\mathrm{c})$ and $\gamma_{p}=10 \mathrm{~ns}^{-1}$ (d-f). The normalized current is $\mu=1.1$. The meaning of the symbols is the same as in Fig. 2.

$$
S_{1}=2 \operatorname{Re}\left(E_{+} E_{--}^{*}\right)=\left|E_{x}\right|^{2}-\left|E_{y}\right|^{2}=P \cos (2 \phi) \cos (2 \chi),
$$

$$
S_{2}=-2 \operatorname{Im}\left(E_{+} E_{-}^{*}\right)=-2 \operatorname{Re}\left(E_{x}^{*} E_{y}\right)=P \sin (2 \phi) \cos (2 \chi),
$$

$$
S_{3}=\left|E_{+}\right|^{2}-\left|E_{-}\right|^{2}=-2 \operatorname{Im}\left(E_{x}^{*} E_{y}\right)=P \sin (2 \chi) .
$$

For polarized light the relation $S_{0}^{2}=S_{1}^{2}+S_{2}^{2}+S_{3}^{2}$ is fulfilled all the time. Therefore $S_{j} / S_{0}$ for $j=1,2,3$ describes the Cartesian components of a unit sphere. The spherical coordinates are the polarization angle $2 \phi \in[0,2 \pi]$ and the ellip-

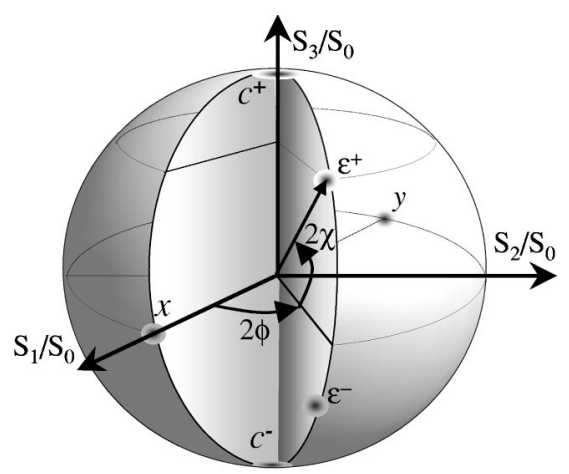

FIG. 4. Poincaré sphere: $x(y)$ LP state along the $\hat{x}(\hat{y})$ direction; $c^{ \pm}$are right and left circularly polarized states, $\varepsilon^{ \pm}$are right and left elliptically polarized states. Shaded circles represent fluctuations around these states. ticity angle $2 \chi \in[-\pi / 2, \pi / 2]$. An alternative description of the electrical fields is in terms of the polarization orientation and ellipticity angles

$$
E_{ \pm}=\sqrt{P / 2}[\cos \phi \mp i \sin \phi][\cos \chi \pm \sin \chi] e^{-i \varphi_{0}},
$$

while the linear components read

$$
\begin{aligned}
& E_{x}=\sqrt{P}[\cos \chi \cos \phi-i \sin \chi \sin \phi] e^{-i \varphi_{0}}, \\
& E_{y}=\sqrt{P}[\cos \chi \sin \phi+i \sin \chi \cos \phi] e^{-i \varphi_{0}},
\end{aligned}
$$

$\varphi_{0}$ being an arbitrary phase. We linearize Eqs. (13b)-(13d) around an $\hat{x}$-LP solution, i.e., $\phi \ll 1$ and $\chi \ll 1$, and assuming constant total intensity $[P(t) \approx \bar{P}]$ we obtain

$$
\delta\left(S_{3} / S_{0}\right) \approx 2 \delta \chi \approx 2 \delta P_{ \pm} / \bar{P}
$$

Equation (16) reveals the connection between the ellipticity fluctuations described in Ref. [18] and the notation used in this paper in terms of power fluctuations of the circular components. Hence, fluctuations of the circular components are linked to movements orthogonal to the equatorial plane of the Poincare sphere.

When analyzing the polarization state evolution on the Poincare sphere, we observe that for small $\gamma_{p}$ the fluctuations prefer the equatorial direction [Fig. 3(c)]. In the limiting case of $\gamma_{p}=0$ the polarization diffuses along LP states with different orientation angles due to the presence of noise. The polarization evolves along the equator of the sphere with small ellipticity fluctuations controlled by $\gamma_{s}$ [Fig. 2(c,f)]. For $\gamma_{p}=0$ there is a zero eigenvalue associated with the arbitrariness of the polarization orientation. When $\gamma_{s}$ is large, the two remaining eigenvalues become real. One of them approaches zero when $\gamma_{s} \rightarrow \infty$, describing diffusion of the ellipticity angle. In the latter, there is no preference for any polarization state and the fluctuations cover the whole surface of the Poincare sphere [38]. When $\gamma_{p} \neq 0$, the eigenvalue that describes the diffusion of the polarization orientation angle becomes nonzero, providing the stability of the steady state. For moderate to large values of $\gamma_{p}$, we observe that the polarization orientation is fixed and the fluctuations on the Poincare sphere have a rather circular shape [Fig. $3(\mathrm{f})]$.

\section{POLARIZATION ANTICORRELATIONS}

To better characterize the correlations between two orthogonal components we compute the normalized crosspower spectral density [21] which reads

$$
C_{A B}(\omega)=\frac{S_{A+B}(\omega)-S_{A}(\omega)-S_{B}(\omega)}{2 \sqrt{S_{A}(\omega) S_{B}(\omega)}},
$$

where $A(t)$ and $B(t)$ represent two given signals. $C_{A B}(\omega)$ $=1(-1)$ corresponds to perfect correlation (anticorrelation) in the fluctuations of the two signals. The normalized cross- 


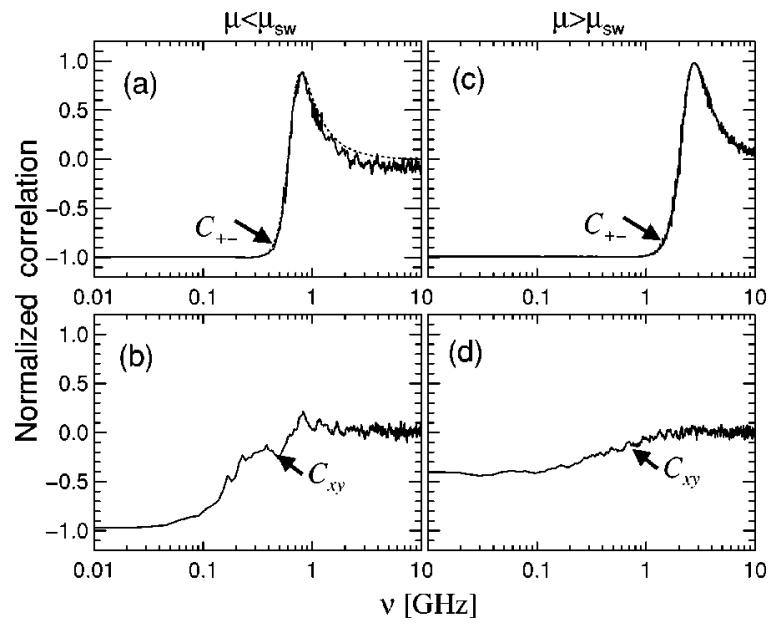

FIG. 5. Normalized cross correlation of the circular components $C_{+-}(\mathrm{a}, \mathrm{c})$ and of the linear components $C_{x y}(\mathrm{~b}, \mathrm{~d})$ corresponding to the same situation as in Fig. 1. Dotted lines in $(a, c)$ represent theoretical prediction given by Eq. (18).

power spectral density between the power fluctuations of the two circular components $\delta P_{+}$and $\delta P_{-}$can be obtained from

$$
C_{+-}(\omega)=\frac{S_{P}(\omega)-S_{q}(\omega)}{S_{P}(\omega)+S_{q}(\omega)} .
$$

On replacing the expression for $S_{P}(\omega)$ and $S_{q}(\omega)$ from Eqs. (11a) and (11b) into Eq. (18) we obtain the exact expression for $C_{+-}$. This expression can be simplified at low frequencies in the case of close to perfect anticorrelation, i.e., $C_{+-}(\omega \approx 0) \approx-1$, to

$$
C_{+-}(\omega=0) \approx-1+\frac{1}{2 Q^{4}} \frac{\left[\left(\gamma_{p} / \kappa\right) \Gamma-\varepsilon \alpha\right]^{2}}{\left[\alpha^{2}+\Gamma^{2}\right]}
$$

with $\Gamma \equiv \gamma_{s} /\left(2 \gamma Q^{2}\right)$. It is easy to see from Eq. (18) that the fluctuations are correlated at the frequency where $S_{P}(\omega)$ $\gg S_{q}(\omega)$, i.e., close to $\Omega_{R}$, and the fluctuations are anticorrelated when $S_{P}(\omega) \ll S_{q}(\omega)$, near the CO peak $\Omega_{P}$. We can interpret these two limits with the help of the Poincare sphere. The fluctuations of the total number of photons are linked to movements toward the inside and outside of the Poincaré sphere surface (Fig. 4). These movements are equivalent for all the points on the sphere, and therefore provide correlation between circular components. Anticorrelations between components are associated with movements on the Poincaré sphere, i.e., polarization orientation and ellipticity fluctuations.

In Fig. 5 we show the results for the normalized crosscorrelation function, Eq. (18), under the same conditions as in Fig. 1. In the circular basis $C_{+-}$, we find close to perfect anticorrelations for low frequencies, and strongly correlated fluctuations $\left(C_{+-} \approx 1\right)$ for frequencies close to the RO peak.
In the linear basis, $C_{x y}$ displays partially anticorrelated fluctuations at low frequency $(\nu \leqslant 1 \mathrm{GHz})$ due to PPN $[1,39]$ and large anticorrelation below PS, which corresponds to the two LP states being stable. The lack of anticorrelation above PS might be attributed to the modification of the effective birefringence due to the nonlinearities when the injection current is increased. Hence, below the PS, the effective birefringence $\Omega_{P} / \pi$, given by Eq. (5), reaches a minimum. This fact leads to preferential fluctuations of the polarization orientation (Sec. IV) and anticorrelated fluctuations of the linear components. On the contrary, above the PS, the effective birefringence gradually increases with increasing distance from the PS, leading to a reduction of the anticorrelation.

\section{CONCLUSIONS}

We presented analytical and numerical investigations of the polarization resolved power spectra of VCSELs operating in the fundamental transverse mode. Our semiclassical framework is valid for arbitrary values of the spin flip rate and birefringence. We presented specific results for the power spectra of linearly polarized states when the VCSEL is driven across a nonthermal polarization switching. The power spectrum of the total intensity fluctuations displays a peak at the relaxation oscillation frequency. The power spectra of the two circular components coincide and show an additional peak associated with coupled oscillations of the ellipticity and polarization orientation angles. Analyzing the evolution of the polarization state on the Poincare sphere, we have been able to separate the effects of the birefringence and spin flip rate. In the absence of birefringence, the spin flip rate controls the ellipticity fluctuations that are related to the fluctuations of the circular components. On the other hand, for a fixed spin flip rate the birefringence controls the polarization orientation fluctuations. The frequency dependence of the normalized cross-correlation function for both linear and circular components has been presented. In particular, the two circular components are strongly anticorrelated for low frequencies while close to perfect correlation is observed near the relaxation oscillation peak. The linear components (lasing and nonlasing) present a nonvanishing cross-correlation function at low frequencies. Linear components display partially anticorrelated fluctuations below the polarization switching, which correspond to the two linearly polarized states being stable. Further investigations of the polarization resolved power spectra of elliptical and dynamical states, where the total intensity and polarization fluctuations do not decouple, should provide a more complete understanding of the noise properties of vertical-cavity surfaceemitting lasers.

\section{ACKNOWLEDGMENTS}

This work has been funded by the European Commission through the VISTA HP-TRN and the Spanish MCyT under Projects No. TIC99-0645-C05-02 and No. BFM2000-1108. Authors also acknowledge fruitful discussions with S. Balle. 
[1] G.P. Agrawal, Phys. Rev. A 37, 2488 (1988).

[2] F. Koyama, K. Morito, and K. Iga, IEEE J. Quantum Electron. 27, 1410 (1991).

[3] K.H. Hahn, M.R. Tan, and S.-Y. Wang, Electron. Lett. 30, 139 (1994).

[4] D.V. Kuksenkov, H. Temkin, and S. Swirhun, Appl. Phys. Lett. 67, 2141 (1995).

[5] D.C. Kilper, P.A. Roos, J.L. Carlsten, and K.L. Lear, Phys. Rev. A 55, 3323 (1997).

[6] J.-L. Vey and W. Elsässer, J. Opt. Soc. Am. B 14, 1299 (1997).

[7] J.-L. Vey and W. Elsässer, Opt. Lett. 23, 721 (1998).

[8] F. Marin and G. Giacomelli, J. Opt. B: Quantum Semiclassical Opt. 1, 128 (1999).

[9] G. Giacomelli, F. Marin, M. Gabrysch, K.H. Gulden, and M. Moser Opt. Commun. 146, 136 (1998).

[10] J.P. Hermier, A. Bramati, A.Z. Khoury, E. Giacobino, J- Ph. Poizat, T.J. Chang, and P. Grangier, J. Opt. Soc. Am. B 16, 2140 (1999).

[11] J.P. Hermier, A. Bramati, A.Z. Khoury, V. Josse, E. Giacobino, P. Schnitzer, R. Michalzik, and K.J. Ebeling, IEEE J. Quantum Electron. 37, 87 (2001).

[12] A. Valle, J. Sarma, and K.A. Shore, IEEE J. Quantum Electron. 31, 1423 (1995).

[13] A. Valle and L. Pesquera, Proc. SPIE 3625, 46 (1999).

[14] J.Y. Law and G.P. Agrawal, IEEE Photonics Technol. Lett. 9, 437 (1997).

[15] M. San Miguel, in Semiconductor Quantum Optoelectronics, edited by A. Miller, M. Ebrahimzadeh, and D.M Finlayson (Institute of Physics, Bristol, 1999), p. 339.

[16] K.J. Ebeling, in Semiconductor Quantum Optoelectronics (Ref. [15]), p. 295.

[17] M.P. van Exter, M.B. Willemsen, and J.P. Woerdman, Proc. SPIE 3946, 58 (2000).

[18] M.P. van Exter, M.B. Willemsen, and J.P. Woerdman, Phys. Rev. A 58, 4191 (1998).

[19] M.P. van Exter, A. Al-Remawi, and J.P. Woerdman, Phys. Rev. Lett. 80, 4875 (1998).

[20] M.B. Willemsen, M.P. van Exter, and J.P. Woerdman, Phys. Rev. A 60, 4105 (1999).

[21] E. Goodbar, J.W. Scott, B. Thibeault, G. Robinson, Y. Akulova, and L.A. Coldren, Appl. Phys. Lett. 67, 3697 (1995).
[22] C. Degen, J.-L. Vey, P. Schnitzer, and K.J. Ebeling, Electron. Lett. 34, 1585 (1998).

[23] M.P. van Exter, M.B. Willemsen, and J.P. Woerdman,J. Opt. B: Quantum Semiclass. Opt. 1, 637 (1999).

[24] M. Giudici, T. Ackemann, S. Barland, J. Tredicce, and S. Balle, J. Opt. Soc. Am. B 16, 2114 (1999).

[25] J.-P. Hermier, I. Maurin, A. Bramati, A.Z. Khoury, and E. Giacobino (unpublished).

[26] M. San Miguel, Q. Feng, and J.V. Moloney, Phys. Rev. A 52, 1728 (1995).

[27] T. Erneux, J. Danckaert, K. Panajotov, and I. Veretennicoff, Phys. Rev. A 59, 4660 (1999).

[28] M.B. Willemsen, M.P. van Exter, and J.P. Woerdman, Phys. Rev. Lett. 84, 4337 (2000).

[29] J. Martin-Regalado, F. Prati, M. San Miguel, and N.B. Abraham, IEEE J. Quantum Electron. 33, 765 (1997).

[30] J. Martin-Regalado, J.L. Chilla, J.J. Rocca, and P. Brusenbach, Appl. Phys. Lett. 70, 3350 (1997).

[31] S. Balle, E. Tolkachova, M. San Miguel, J. Tredicce, J. MartinRegalado, and A. Gahl, Opt. Lett. 24, 1121 (1999).

[32] K.D. Choquette, D.A. Richie, and R.E. Leibenguth, Appl. Phys. Lett. 64, 2062 (1994).

[33] B. Ryvkin, K. Panajotov, A. Georgievski, J. Danckaert, M. Peeters, G. Verschaffelt, H. Thienpont, and I. Veretennicoff, J. Opt. Soc. Am. B 16, 2106 (1999); K. Panajotov, B. Ryvkin, J. Danckaert, M. Peeters, H. Thienpont, and I. Veretennicoff, IEEE Photonics Technol. Lett. 10, 6 (1998).

[34] A. Valle and L. Pesquera, IEEE Photonics Technol. Lett. 13, 272 (2001).

[35] M. San Miguel and R. Toral, in Proceedings of Instabilities and Nonequilibrium Structures VI, edited by E. Tirapegui, J. Martinez, and R. Tiemann (Kluwer Academic, Dordrecht, 2000), p. 35; C.W. Gardiner, Handbook of Stochastic processes 2nd ed. (Springer-Verlag, Berlin, 1995).

[36] R.F.M. Hendriks, M.P. van Exter, J.P. Woerdman, L. Weegels, and A. Van Geelen, J. Opt. Soc. Am. B 16, 832 (1999).

[37] M. San Miguel, S. Balle, J. Mulet, C.R. Mirasso, E. Tolkachova, and J.R. Tredicce, Proc. SPIE 3944, 242 (2000).

[38] S. Ciuchi, M. San Miguel, and N.B. Abraham, Phys. Rev. A 57, 3843 (1998).

[39] J.-L. Vey, C. Degen, K. Auen, and W. Elsässer, Phys. Rev. A 60, 3284 (1999). 